\title{
LA CATASTROPHE RADIOACTIVE DE GOIÂNIA AU BRÉSIL
}

Conflits sur l'interprétation d'un désastre, comment vivre après ?

Telma Camargo da Silva

Association Multitudes | « Multitudes »

2015/1 n $58 \mid$ pages 161 à 166

ISSN 0292-0107

Article disponible en ligne à l'adresse :

https://www.cairn.info/revue-multitudes-2015-1-page-161.htm

Distribution électronique Cairn.info pour Association Multitudes.

(C) Association Multitudes. Tous droits réservés pour tous pays.

\section{(cc) BY-NC}




\section{La catastrophe radioactive de Goiânia au Brésil Confllits surir l'interprétation d'uin désastire, comment vivire après? Telma Camargo da Silva}

Fin février 2014, la presse mondiale annonçait une nouvelle fuite d'eau contaminée de la centrale Fukushima Daiichi ravagée par le tsunami du 11 mars 2011. Selon l'opérateur Tokyo Electric Power (Tepco), cette eau, bien que débarrassée de césium radioactif, contient encore des radionucléides qui émettent des rayons bêta. Le porte-parole de la centrale déclara lors d'une conférence de presse que la fuite d'eau ne s'est probablement pas propagée jusqu'à l'océan Pacifique. À l'instar d'autres désastres radioactifs, les experts essayent de contenir le péril nucléaire en contrôlant la représentation du risque radioactif: le fossé du réservoir est loin de la mer...; la dose de l'exposition à la radiation atteint des limites tolérables...; les conséquences de la radiation sont objectivement fixées dans le temps et dans espace...; la fin du désastre est déterminée par la fin du processus de décontamination.

Or, les expériences rapportées par les survivants des désastres radioactifs comme ceux de Chernobyl (1986, ex URSS) et Goiânia (1987, Brésil) ou des essais nucléaires en Polynésie française (1966-1996) dévoilent les dimensions politiques de ces interprétations. Les conséquences réelles des doses de radiation sont cachées, ou les déclarations des experts sont modifiées en réponse aux demandes d'éclaircissements et de justice faites par des groupes organisés. Les désastres et leurs conséquences se prolongent tant que les conflits sur leurs interprétations persistent.

Ainsi à Goiânia, vingt-sept ans après l'ouverture d'un appareil abandonné de radiothérapie contenant du césium-137, des groupes de survivants militent toujours en vue de la reconnaissance de leur statut de victimes. L'Association des Victimes du Cesium-137 demande à l'État l'assurance de soins médicaux aux victimes légalement reconnues. Des secteurs organisés de la société civile persistent avec leur projet de bâtir un musée du désastre ${ }^{1}$. Ce

1 Une version de cet article a été présentée dans le Séminaire Internationale TransOceanik, Florianópolis (Brésil), Mai 2013. Sur ce musée, voir mon article: «Musealização de eventos críticos: análise da tensão entre múltiplas narrativas de dor». In: TAMASO, Izabela; LIMA FILHO, Manuel Ferreira. Antropologia e Patrimônio Cultural. Brasília: ABA. 2012, p. 497-525. 
travail de la mémoire 2 , sujet de mes recherches, en déployant le processus de fabrication sociale du désastre au-delà de l'interprétation du seuil de cessation d'activité proposée par les experts nucléaires, révèle que les technologies développées au $\mathrm{XX}^{\mathrm{e}}$ siècle ont créé de nouveaux critères pour penser les rapports sociaux et les identités sociales.

\section{Scénarios vs. scènes}

L’analyse du désastre radioactif de Goiânia ${ }^{3}$ fournit des éléments pour penser d'autres désastres dont la souffrance sociale ${ }^{4}$ est engendrée par les conflits d'interprétation de l'identité de victime en fonction de la dose de radiation: conçue comme objective selon les experts nucléaires, cette identité est en fait déclinée de manière subjective comme le montre l'analyse des désastres. Pour situer les pratiques des acteurs sociaux sur les lieux contaminés par la radiation, j'adopte le concept de "scénario", en portugais cenário, proposé par José Guilherme Magnani ${ }^{5}$. Contrairement au sens courant de ce terme, qui renvoie aux actions se déroulant selon un plan préétabli et immobile, un scénario résulte ici des pratiques sociales en mouvement ayant lieu sur un endroit précis. Les expériences vécues au passé sont mises en dialogue avec celles du présent de manière à ce

2 Je me rapporte à la notion proposée par Elizabeth Jelin (Los Trabajos de la memoria. Madrid: Siglo XXI de España Editores and Social Sciences Research Council. 2002) selon laquelle les souvenirs sont actualisés par les rapports établis entre différents acteurs sociaux.

3 Je me permets de renvoyer aux réflexions faites auparavant dans mon livre Radiation Narratives and Illness: the Politics of Memory on the Goiannia Disaster, VDM Verlag Dr. Muller, Saarbrucken, 2009.

4 Das, V., Kleinman, A., Lock, M. (Éds), 1998. Social Suffering, Oxford University Press, Delhi.

5 José Guilherme Magnani, L. de L. Torres, «Quando o campo é a cidade - fazendo antropologia na metrópole», Na metrópole, São Paulo, Editora da USP, FAPESP, 1996, p. $12-53$. que les expériences du présent puissent confirmer ou contester celles du passé. Donc le scénario est déterminé par les usages de ses acteurs et il n'est jamais le même. Cette expérience du corps vécue sur un endroit, resignifiée par l'acte de narrer, est décrite comme place embodied par Edward Casey. ${ }^{6}$ Les deux concepts parlent d'une «scénarisation » du désastre par les voix des victimes qui rendent actuels les faits de 1987.

Par contraste avec la territorialisation dynamique qu'illustre la notion de «scénarisation", l'expertise nucléaire renvoie plutôt à l'idée d'une scène du désastre, dont l'événement et les personnages sont fixés au passé. Scénario et scène en tant que concepts condensent aussi le conflit sur la reconnaissance de la victime du désastre. Dans ce sens, je soutiens que lieu, corps, mémoire et narration sont inter-reliés dans la production des identités de victime du désastre radioactif de Goiânia.

Les conflits d'interprétation sur les doses de radiation configurent la dimension politique de la gestion des désastres radioactifs et de leurs réponses. Ils signalent la censure des problèmes vécus par les survivants et l'invisibilisation de leurs problèmes de santé et de souffrance, que les mouvements de résistances tentent de rendre visibles. Les impacts sociaux des désastres nucléaires continuent ainsi bien au-delà de l'événement. L'exemple brésilien de Goiânia souligne l'actualité transnationale du fait que ces désastres sont à la croisée d'enjeux juridiques, technologiques et environnementaux, où la perception du risque, et la gestion gouvernementale se confrontent à la santé, à la résilience et aux voix des victimes.

6 Steven Feld, «How to get from space to place in a fairly short stretch of time». in Keith Basso, Senses of Place, Santa Fé, School of American Research Press,1996, p. 13-52. 
Les scénarios

de la technologie nucléaire et l'illusion de l'objectivité

La configuration de la scène de la technologie nucléaire se fait d'après $1^{\circ}$ le contrôle objectif de la radiation et $2^{\circ}$ l'invention d'équipements qui matérialisent la radiation, cet élément immatériel, sans odeur, sans couleur et silencieux. Cette technologie est basée sur le concept que la radioactivité est objectivement accessible par des instruments pleinement fiables. C'est la confiance pleine dans la technologie qui assure au système nucléaire la croyance dans les limites tracées pour les risques de contamination.

Pour les habitants d'une ville vivant un désastre radioactif, c'est la confiance dans les actions entreprises par l'expertise nucléaire qui permet de surmonter la peur et de continuer à vivre dans une situation de risque. Cette scène, caractérisée par la corrélation entre risque et fiabilité , par la maîtrise d'une nouvelle technologie et par la croyance dans l'objectivité des mesures, confère au système d'expertise nucléaire l'autorité de reconnaître, d'après les critères des biosciences, les nouvelles identités engendrées par l'exposition radioactive: les radioacidentados. ${ }^{8}$

Cependant, la scène du désastre comporte des contradictions et des ambiguïtés qui appellent d'autres définitions. Selon la perspective de la rationalité mathématique, les «zones chaudes» étaient clairement signalées par des pièces de bois rouges qui séparaient

7 Je reprends ici l'idée proposée par Ulrich Beck, Risk Society, Sage Publications, Londres, 1986.

8 Pendant les années 1987-2000, seuls les radioacidentados - porteurs des marques physiques de la radiation - étaient reconnus par l'État comme victimes et donc seuls ils avaient droit aux lois compensatoires. Je pense ici aux «biological citizens» de Adriana Petryna dans, Life Exposed, Princeton University Press, Princeton \& Oxford, 2002. et isolaient les immeubles contaminés par la radiation et dont l'entrée était interdite. Mais, ces points rouges - indices du paysage urbain contaminé - étaient aussi perçus par les habitants comme l'emblème de la contradiction. Beaucoup de zones isolées étaient des rues et les personnes circulaient normalement entre ces bois rouges. Leur expérience mettait en cause les frontières des zones contaminées, et la classification officielle des personnes contaminées. La confiance dans le système d'expertise nucléaire, nécessaire pour surmonter la peur de la contamination radioactive, était détruite par l'expérience de ces contradictions.

\section{Bois rouges et vêtements}

Les bois rouges symbolisaient la séparation entre les «vizinhos de foco» (voisins des hautes doses) et les radioacidentados, et aussi entre les travailleurs nucléaires et les travailleurs nonnucléaires qui sont intervenus au début de la gestion de la catastrophe. Cependant, l'expérience vécue avec le désastre montra une autre réalité, décrite ci-dessus, qui provoqua des soupçons et amena les gens à agir, à travers l'Associação das Vítimas do Césio-137 (L'Association des Victimes du Césium-137) crée en décembre 1987.

Le processus qui donna naissance à l'Association résulte de la demande d'éclaircissement sur les limites des doses de radiation et celles des espaces de circulation des personnes dans les endroits balisés comme contaminés. A aussi participé à ce processus la revendication pour l'inclusion de marques invisibles de la radiation qui permettent de définir le statut de victime - entre autres, la désintégration des réseaux sociaux, les pertes économiques et les troubles émotionnels. L'Association proposa deux catégories de 
victimes: les victimes directes, correspondant à celle de radioacidentados, et les victimes indirectes, comprenant les voisins des points chauds. L'Association tire donc son origine du processus d'organisation des voisins qui s'identifient comme des victimes indirectes et se trouvent pris entre une identité attribuée et une identité revendiquée.

Cette identité engendrée par l'activisme caractérise aussi celle des polices militaires - os policiais do césio, comme la presse de Goiânia les a nommées. Au début des années 1997, dix ans après l'ouverture de l'appareil de radiothérapie, ces policiers racontaient dans les media leurs propres problèmes de santé, perçus comme "maladies inexpliquées». Leurs témoignages avaient un point en commun: le travail de surveillance réalisé au Depósito Provisório de Rejeitos Radioativos de Abadia de Goiás - Dépôt Provisoire des Déchets Radioactifs de Abadia de Goiás - et/ou, en 1987, dans les endroits indiqués par la Comissão Nacional de Energia Nuclear-CNEN - comme des points chauds et signalés par les bois rouges.

Face à l'expérience de la maladie, ces officiers rappellent le travail fait sur un lieu considéré comme contaminé. En cherchant dans le passé les souvenirs de leurs activités professionnelles, ils rendaient compte de la distinction entre professionnels nucléaires et non-nucléaires travaillant dans la réponse au désastre radioactif. Cette comparaison mettait en évidence l'absence de vêtements et d'équipements de protection pour travailler sur la scène du nucléaire.

La construction de la causalité entre travail - maladie - radiation souligne une idée récurrente dans leurs témoignages: ils ont été contaminés parce qu'ils n'ont pas été informés ni équipés pour travailler dans une zone radioactive ${ }^{9}$. C'est la perception de deux corps différents travaillant dans la réponse au désastre - le corps de l'agent de police et celui de l'expert nucléaire - qui provoque des angoisses concernant leurs santés.

Cette perception est signalée, en premier lieu, par le vêtement. Les policiers étaient malades parce qu'ils ne portaient pas les combinaisons utilisées par les travailleurs nucléaires. En fait, les experts nucléaires avec leurs combinaisons en couleur, blanche, orange ou brune ont provoqué un impact dans l'imagination du peuple de la ville, y compris les policiais do césio. Associés à la CNEN - objet de méfiance et de critique - ces vêtements signifiaient en même temps le travail de décontamination. Ils sont devenus, pour cette raison, le symbole d'une contradiction: rappel de l'expertise et de l'autorité de ces techniciens, mais aussi de la méfiance par rapport aux doses de radiation et à la circulation aux alentours des bois rouges.

En 1997, ces combinaisons apparaissent dans les témoignages des policiais do césio comme un des éléments indicatifs de la différence entre deux groupes de professionnels. Par effet métonymique, elles sont à mon avis une manière de parler des sociabilités, de «l'embodiment», et des identités des travailleurs dans le contexte du désastre radioactif de Goiânia. Fondé sur la compréhension de la nature morale du tissu et du vêtement - avec sa capacité à incorporer et à transmettre des

9 Similaire aux témoins des essais nucléaires français (1966-1996) en Polynésie. Les jeunes Polynésiens engagés avec les jeunes de métropole en service militaire pour nettoyer le site à chaque explosion ne portaient pas de tenues de protection spéciales. Comme pour les policiais do césio, la prise de conscience de la contamination radioactive s'est faite lentement. Bruno Barrillot, Marie-Hélène Villierme et Arnaud Hudelot, Témoins de la Bombe, Univers Polynésiens, Tahiti, 2012, livre et DVD de l'exposition. Voir aussi www.mururoa.org 


\section{Hors-Champs La catastrophe radioactive de Goiâna}

valeurs sociales ${ }^{10}$ - l'uniforme porté par les travailleurs nucléaires a été transformé en signe politique. D'un côté, il légitime et donne autorité aux experts nucléaires. De l'autre, le système nucléaire nie l'identité de victime des policiais do césio, en refusant leur argument quant à leur droit à l'usage de ces vêtements.

\section{Mémoire incarnée, mémoire performative}

À défaut de marques visibles de la radiation - les radiolésions - et d'enregistrement des doses subies, les agents de police décident en 1997 de rendre concrète leur «appartenance» au désastre en exposant leurs corps et les images de leurs radiographies. Pour rendre visible l'invisibilité des marques, ils exhibent - pour que les médias puissent les enregistrer - leurs corps malades: la tête rasée, suite à une chirurgie pour enlever une tumeur; la perte des cheveux due à la radiothérapie; les tâches sur les corps. J'interprète ces performances - l'exhibition publique du corps en souffrance - comme la mémoire incarnée ${ }^{11}$ des survivants qui souligne le lieu et le corps comme des éléments constitutifs de l'identité de victime: le récit sur l'expérience d'avoir été là - indépendamment de l'existence de la documentation sur les doses de contamination radioactive. Ainsi, la mémoire performative $^{12}$ s'oppose aux récits des experts nucléaires qui dénient la souffrance des soldats travaillant durant la première étape de la gestion du désastre.

10 Roland Barthes, Système de la mode, Seuil, Paris, 1967. Annette Weiner, Jane Schneider, Cloth and Human Experience, Smithsonian Institution Press, Washington et Londres, 1989.

11 Voir le concept de bodily memory de Marita Sturken, Tangled Memories, Basic Books, New York, 1996.

12 Paul Connerton, How societies remember, Cambridge University Press, Cambridge, 1996.
Au fil des années, la classification de victime dévoile deux mouvements opposés. D'une part, des mesures prises pour limiter le nombre de personnes blessées. D'autre part, des mobilisations vers la reconnaissance. Ce conflit d'interprétations n'est pas nouveau et il révèle que les expériences subjectives des survivants contribuent à la compréhension de la corrélation existant entre les dimensions biologiques, légales, politiques et sociales propres aux évènements critiques. ${ }^{13}$ En même temps, cela rend visible l'organisation face au système bio-nucléaire.

\section{Le silence comme résistance} Cependant, il y a une autre identité parallèle à cette identité recherchée, que je viens d'analyser. Les fils et filles de victimes classés comme radioacidentados - eux aussi radioacidentados et qui étaient enfants en 1987 - cachent cette identité et leurs histoires de famille. Les récits que m'ont apportés ces jeunes révèlent l'existence d'une douleur engendrée par le paradoxe entre l'affirmation d'une identité tracée par l'expérience et désignée par le système d'expertise, alors que l'invisibilité se poursuit en tant que survivants du désastre. Les subjectivités de ces jeunes sont entrelacées au désastre dont les remémorations actualisent la tragédie dans leur vie quotidienne: les marques faites par la brûlure de la radiation sur la peau ; l'odeur du vinaigre employé pour laver le corps et retirer l'élément radioactif de la peau. Tout cela évoque une souffrance qu'ils ne peuvent pas surmonter. Les souvenirs, les représentations et les sensations

13 En ce qui concerne la perception de la santé associée à ce processus, voir notamment Veena Das, "Suffering, Legitimacy and Healing», Critical Events, Oxford University Press, Nova Delhi, 1996, p. 135-174. 
vécues par le corps font partie de la construction du silence et engendrent l'identité cachée des jeunes survivants.

Cette deuxième identité, comme l'a bien signalé Robert J. Lifton ${ }^{14}$ à propos d'Hiroshima, tire son origine de la situation traumatique résultant de la rencontre avec la mort, rencontre qui n’arrive pas à être réintégrée dans le présent. Les jeunes survivants vivent le paradoxe de deux identités dissociées et les souvenirs ainsi produits constituent la mémoire traumatique. Réminiscences fixées dans un lieu et dans un temps du passé, associées à une expérience de perte, de mort physique et symbolique qui n'ont pas été surmontées au présent, avec pour résultat une tension entre l'expérience passée et la vie à vivre au futur. Pour certains, ce silence représente aussi une manière de résistance, une autre expression de l'agencement face à la rupture provoqué par la catastrophe ${ }^{15}$. Pour les jeunes radioacidentados cacher leurs identités des voisins, des camarades à l'école et au travail signifie aussi n'être plus stigmatisés ${ }^{16}$ à cause de la contamination radioactive dont ils ont souffert en 1987 - et permettre de se construire une nouvelle identité.

\section{Classifications objectives et expériences subjectives}

Dans cette analyse, j'avance que la configuration de multiples identités apportées par les récits construits par les survivants du désastre de Goiânia signale que la maladie et la souf-

14 Death in Life, Random House, New York, 1967.

15 Les processus d'agency dans la souffrance et de création face au désastre sont les sujets du livre dirigé par Barbara Glowczewski, Alexandre Soucaille, Désastre, Paris, L'Herne, Collection Cahiers d'Anthropologie Sociale, 2011.

16 À Goiânia, certaines personnes croient les radioacidentados dangereux parce qu'ils seraient encore en 2013 contaminés par la radiation. france se produisent non seulement dans le corps mais dans «un corps qui est dans le monde ${ }^{17}$ En même temps que les classifications du système bio-nucléaire restreignent la quantité de victimes et encadrent l'événement dans le passé, les expériences subjectives dévoilées par le processus narratif vont au-delà des frontières définies par la biomédecine et encadrent le désastre de Goiânia dans le présent. Les victimes rendues invisibles par l'État résistent aux critères mathématiques de définition et proposent une forme d'identification qui relève d'une territorialisation dynamique. D'autres cachent leur appartenance au désastre comme une forme de résistance. Les uns et les autres dévoilent l'agentivité des hommes et des femmes ${ }^{18}$ face à la contamination radioactive et à la souffrance. Cela montre que la résistance naît de l'action des individus et des groupes envers les différentes classifications dominantes à un certain moment de l'histoire du désastre: l'expertise nucléaire, la connaissance médicale; les institutions gouvernementales; les autorités judiciaires; les habitants non-contaminés de Goiânia; les secteurs économiques de la région. Ce sont ces interfaces troubles entre interprétations bio-scientifiques et expériences subjectives de la souffrance qui rendent visibles le processus d'organisation entrepris par les individus pour récupérer un projet de vie après l'expérience avec la mort et pour surmonter la peur de la contamination radioactive et de ses conséquences.

17 Byron J. Good, Medicine, Rationality and Experience, Cambridge University Press, Cambridge, 1994.

18 Barbara Glowczewski, Alexandre Soucaille, ibid. 